\section{Prevalência de sibilância e fatores de risco associados em crianças no primeiro ano de vida, residentes no Município de Recife, Pernambuco, Brasil}

\author{
Prevalence of wheezing and associated risk factors \\ among infants in Recife, Pernambuco State, Brazil
}

Decio Medeiros 1 Almerinda Rêgo Silva 1 José Ângelo Rizzo 1 Emanuel Sarinho 1 Javier Mallol 2 Dirceu Solé 3

\footnotetext{
1 Universidade Federal de Pernambuco, Recife, Brasil.

2 Universidad de Santiago de Chile, Santiago, Chile.

3 Universidade Federal de São

Paulo, São Paulo, Brasil.

Correspondência

D. Medeiros

Universidade Federal de

Pernambuco.

Av. Professor Morais Rêgo s/n,

Recife, PE 50670-320, Brasil.

daph@elogica.com.br
}

\section{Abstract}

The aim of this study was to verify the prevalence of wheezing in infants (< 1 year of age) in Recife, Pernambuco State, Brazil, and to identify associated risk factors. Sample and methods: the study was performed according to the protocol of the International Study of Wheezing in Infants (EISL) in children ranging from 12 to 15 months of age. The sample was analyzed for presence or absence of wheezing. A total of 1,071 parents of children ranging from 12 to 15 months of age were interviewed. Prevalence of wheezing in the first year of life was 43\%, with no difference between the sexes. Wheezing in the first year of life was associated with pneumonia, family history of asthma, more than nine episodes of upper airway infection, and the first cold before six months of age ( $p<$ 0.001). Prevalence of wheezing in the first year of life was high in Recife. Early onset (and high number) of colds, family history of asthma, and pneumonia were associated with wheezing in these children.

Respiratory Sounds; Asthma; Infant; Risk Factors

\section{Introdução}

Crises de sibilância ou chiado são comuns nos primeiros anos de vida e tendem a decrescer com a idade 1. Estudos epidemiológicos demonstraram que episódios de sibilância precoce e recorrente são associados ao desenvolvimento de asma posteriormente, em idade escolar 2,3 .

Embora a maioria dessas crianças apresente sibilância em fase precoce da vida, estima-se que cerca de $40 \%$ continuarão sibilando aos seis anos de idade 3 . Estudo documentou serem $44 \%$ as crianças menores de um ano de vida atendidas por sintomas respiratórios em unidades de emergência 4. Outros, demonstraram taxas elevadas de hospitalização para crianças menores de um ano com sintomas respiratórios, além de verificarem taxas de hospitalização até três vezes maiores que as de crianças que não apresentavam sibilância 5 . Tais fatos determinaram custos elevados ao sistema de saúde, bem como maior perda de dias de trabalho pelos cuidadores, além de queda na qualidade de vida do infante e de sua família 6 .

A demonstração de que sintomas relacionados à obstrução recorrente de vias aéreas inferiores têm início em idade precoce e que tendem a persistir ao longo da infância em determinadas crianças, leva-nos pensar que o tratamento profilático deva ser iniciado o mais cedo possível.

Um grande dilema para o profissional de saúde é determinar qual criança que apresenta de- 
terminados fatores de risco para persistência de sibilância deverá iniciar tratamento profilático precoce ou não, visto que muitas destas crianças podem apresentar remissão espontânea do quadro de sibilância no futuro ${ }^{3}$.

Estudos demonstraram diversos fatores de risco para sibilância precoce em crianças e sua relação com o posterior desenvolvimento de asma, como: prematuridade 7; tabagismo materno na gestação ou passivo após o nascimento 8,9 ; sexo masculino 9,10; infecções respiratórias 9,11, notadamente pelo vírus respiratório sincicial 12; história familiar de asma ou antecedente pessoal de dermatite atópica ou de rinite 9,13. Também foi demonstrada relação entre sibilância precoce e sensibilização precoce a alimentos ou aeroalérgenos, função pulmonar reduzida e nível sanguíneo elevado de eosinófilos, com posterior persistência destes sintomas 3,14 . Porém, nenhum desses fatores, genéticos ou ambientais, quer façam parte da anamnese, do exame físico ou de meios complementares de diagnóstico, apresentou poder suficiente para ser usado como marcador único na identificação de asma no futuro. Apesar disso, poucos são os estudos que avaliam fatores de risco para sibilância em crianças no primeiro ano de vida 14,15 .

Este estudo foi conduzido com o objetivo de verificar a prevalência de sibilância em lactentes da cidade de Recife, Pernambuco, Brasil, avaliar a possível interferência do sexo e identificar quais fatores de risco estão associados a quadros de sibilância no primeiro ano de vida.

\section{Casuística e método}

Esta pesquisa foi realizada segundo o protocolo do Estudio Internacional de Sibilancia en Lactantes (EISL) 16. Pais e/ou responsáveis por crianças com idades entre 12 e 15 meses de vida e em bom estado geral de saúde responderam ao questionário escrito (QE) padronizado (QEEISL) durante o seu atendimento em postos de saúde da cidade de Recife, quando realizavam consultas e/ou imunizações de rotina. O número mínimo de crianças a serem avaliadas foi determinado em 1.000 17. A lista dessas unidades foi fornecida pela Secretaria de Saúde do município, e a escolha foi realizada segundo o preconizado pelo protocolo EISL.

Neste estudo não foram admitidas crianças que vinham sendo acompanhadas por qualquer outra doença crônica. Não foram alocados departamentos de emergência ou serviços de pronto-atendimento.

O instrumento empregado foi o QE-EISL, previamente validado após a sua tradução para o português (cultura brasileira), e foi respondido pelos pais e/ou responsáveis desses lactentes 18,19,20. Além de dados demográficos como peso e estatura de nascimento e na ocasião da inclusão no estudo, o QE-EISL é composto por 45 perguntas sobre a presença e a frequência de chiado ou sibilância no primeiro ano de vida, idade ao primeiro episódio, uso de serviços de emergência ou internamentos por sintomas respiratórios 18 . Além disso, perguntou-se sobre o uso de medicações inaladas para alívio de sintomas e a sua frequência, diagnóstico médico de asma, idade de início e frequência de infecções de vias aéreas superiores, presença de infecção de vias aéreas inferiores, presença individual ou familiar de atopia, tempo de amamentação, vacinações e tempo de aleitamento materno exclusivo ${ }^{18}$. O QE-EISL pesquisa também condições socioambientais, como tabagismo durante a gestação ou o tabagismo passivo após o nascimento, permanência em creches e a partir de que idade, presença de animais domésticos, ou, segundo o respondedor, de poluição ambiental, número de irmãos, nível de escolaridade dos pais e raça 18 .

Todos os participantes foram orientados sobre o caráter da pesquisa, e antes de iniciarem a resposta ao questionário assinaram o Termo de Consentimento Livre e Esclarecido.

Os dados obtidos foram transferidos a um banco de dados fornecido pelos coordenadores gerais do estudo e foram apresentados como porcentagem de respostas afirmativas divididas por sexo. Posteriormente, a amostra foi analisada segundo a presença ou não de sibilância. A resposta afirmativa à pergunta: "Seu bebê teve chiado no peito, bronquite ou sibilâncias nos seus primeiros doze meses de vida?", identificou os com sibilância que foram selecionados para análise dos fatores de risco para sibilância. De acordo com a natureza das variáveis foram usados testes paramétricos e não paramétricos.

Em todas as análises empregou-se a análise univariada para identificar os possíveis fatores de risco a elas associados (teste do qui-quadrado), complementados pela análise de regressão logística para as variáveis que atingiram nível de significância próximo a 0,05 . Os resultados desses modelos foram apresentados sob a forma de odds ratio (OR) e intervalos de $95 \%$ de confiança (IC95\%) 21.

Para a análise dos dados empregou-se o programa estatístico Epi Info 3.4.3 (Centers for Disease Control and Prevention, Atlanta, Estados Unidos) e o SPSS 12.0 (SPSS Inc., Chicago, Estados Unidos).

O estudo foi previamente aprovado pelos Comitês de Ética em Pesquisas do Centro de Ciências da Saúde, Universidade Federal de Pernam- 
buco (protocolo no. 004/06) e da Escola Paulista de Medicina, Universidade Federal de São Paulo (protocolo no. 0657/06).

\section{Resultados}

Foram entrevistados e responderam ao QE-EISL 1.071 pais e/ou responsáveis pelas crianças participantes do presente estudo, durante o período compreendido entre março e dezembro de 2007. Desses, 564 (52,7\%) eram crianças do gênero masculino. A média ( \pm desvio padrão - DP) de idade foi de $13,5( \pm 1,2)$ meses, peso médio de nascimento de $3,7( \pm 0,8) \mathrm{kg}$ e a média de estatura $48,6( \pm 4,2) \mathrm{cm}$. No momento da avaliação, o peso médio foi de $10,4( \pm 1,7) \mathrm{kg}$ e a estatura média 76,1 $( \pm 6,8) \mathrm{cm}$. A prevalência de sibilância aqui observada foi de $43 \%$.

A análise comparativa entre os gêneros segundo as respostas ao QE-EISL revelou diferenças significantes apenas para o número de episódios de chiado no peito no primeiro ano de vida, o diagnóstico médico de asma, tabagismo passivo e hospitalização por bronquite ou pneumonia, que foram mais frequentes entre as do sexo feminino (Tabela 1). O relato de sibilância no primeiro ano de vida foi significantemente associado ao baixo nível de escolaridade materna, ter ido à creche, ter poluição no local em que vive, estar com o calendário vacinal em dia, estar exposta a fumante no domicílio ou durante a gestação, ter tido pneumonia, ter familiares com asma, ter familiares com alergia ou rinite alérgica, ou alergia de pele, ter tido mais de cinco episódios de infecções de vias aéreas superiores e ter sido o primeiro episódio antes de seis meses de via (Tabela 2). Não houve interferência do gênero nessas variáveis estudadas, exceto com relação ao nível de escolaridade materna e uso de corticosteroide oral pelo lactente no primeiro ano de vida, que predominaram entre as lactentes (dados não mostrados).

Tendo-se como base o relato ou não de sibilância nos primeiros doze meses de vida, complementou-se a análise das variáveis aplicandose o modelo de regressão logística, e as significantes são apresentadas na Tabela 3 e na Figura 1. Demonstrou-se, assim, nesta amostra, quais foram os fatores de risco para a sibilância no primeiro ano de vida, como: ter tido pneumonia e também mais de nove episódios de resfriado, em que, quando presentes isoladamente, elevavam o risco em mais de três vezes para ter quadros de sibilância.

\section{Discussão}

Este estudo utilizou o QE-EISL e caracterizou a frequência e os fatores de risco para sibilância no primeiro ano de vida em crianças de 12-15 meses de idade, residentes na cidade de Recife, cidade litorânea, de clima tropical, com pouca variação de temperatura e alta umidade relativa do ar durante o ano. A prevalência de episódios de sibilância no primeiro ano de vida foi de $43 \%$. Dados semelhantes aos observados por Chong et al. ${ }^{21}$ na cidade de Curitiba, Paraná, que utilizaram o mesmo protocolo. Martinez et al. 22, na coorte de Tucson, Estados Unidos, relataram que aproximadamente $34 \%$ das crianças por eles

Tabela 1

Distribuição dos pacientes segundo as respostas ao QE-EISL e o sexo, dados significantes. Recife, Pernambuco, Brasil, 2007.

\begin{tabular}{|c|c|c|c|c|c|c|c|}
\hline \multirow[t]{2}{*}{ Itens } & \multicolumn{2}{|c|}{ Feminino } & \multicolumn{2}{|c|}{ Masculino } & \multicolumn{2}{|c|}{ Total } & \multirow[t]{2}{*}{ Valor de $p$ * } \\
\hline & $\mathrm{n} / \mathrm{N}$ & $\%$ & $n / N$ & $\%$ & $n / N$ & $\%$ & \\
\hline \multicolumn{8}{|c|}{ Episódios de chiado no peito no primeiro ano de vida? } \\
\hline$<3$ & $89 / 241$ & 36,9 & $111 / 225$ & 49,3 & $200 / 466$ & 42,9 & 0,026 \\
\hline$\geq 3$ & $152 / 241$ & $63,1 \star \star$ & $114 / 225$ & 50,6 & $266 / 466$ & 57,1 & \\
\hline Tratamento com corticosteroide oral? & $96 / 216$ & $44,4^{\star \star}$ & $66 / 196$ & 33,7 & $162 / 412$ & 39,3 & 0,025 \\
\hline Lactente foi hospitalizado por bronquite? & $62 / 241$ & $25,7^{\star \star}$ & $34 / 225$ & 15,1 & $96 / 466$ & 20,6 & 0,005 \\
\hline Lactente teve diagnóstico de asma por médico? & $62 / 241$ & $25,7 \star \star$ & $37 / 225$ & 16,4 & $99 / 466$ & 21,2 & 0,014 \\
\hline Lactente foi hospitalizado por pneumonia? & $85 / 564$ & $15,1 \star \star$ & $47 / 507$ & 9,3 & $132 / 1071$ & 12,3 & 0,004 \\
\hline Você fuma? & $99 / 564$ & 17,6 ** & $64 / 507$ & 12,6 & $163 / 1.071$ & 15,2 & 0,025 \\
\hline
\end{tabular}

QE-EISL: questionário escrito padrão do Estudio Internacional de Sibilancia en Lactantes.

* Teste qui-quadrado;

** Valores significantes. 
Fatores associados (variáveis sociodemográficas, comportamentais e clínicas) com a presença $(n=466)$ de sibilância no primeiro ano de vida, variáveis significantes. Recife, Pernambuco, Brasil, 2007.

\begin{tabular}{|c|c|c|c|c|c|}
\hline \multirow[t]{3}{*}{ Variáveis } & \multicolumn{4}{|c|}{ Presença de sibilância } & \multirow[t]{3}{*}{ Valor de $p$ * } \\
\hline & \multicolumn{2}{|c|}{ Sim } & \multicolumn{2}{|c|}{ Não } & \\
\hline & $\mathbf{n}$ & $\%$ & $\mathrm{n}$ & $\%$ & \\
\hline Escolaridade materna & & & & & $<0,001$ \\
\hline Básica, primária ou nenhuma & 184 & 49,9 & 185 & 50,1 & \\
\hline Média ou secundária incompleta & 159 & 45,3 & 192 & 54,7 & \\
\hline Secundária completa ou superior & 123 & 35,0 & 228 & $65,0 * \star$ & \\
\hline Número de irmãos & & & & & 0,057 \\
\hline Nenhum & 161 & 39,3 & 249 & 60,7 & \\
\hline 1 & 139 & 44,3 & 175 & 55,7 & \\
\hline 2 ou mais & 166 & 47,8 & 181 & 52,2 & \\
\hline O lactente foi à creche & 162 & 56,6 ** & 124 & 43,4 & $<0,001$ \\
\hline Há poluição onde vive & 298 & 48,1 & 321 & $51,9 * \star$ & $<0,001$ \\
\hline Esquema vacinal em dia? & 432 & 42,8 & 577 & $57,2 * \star$ & 0,048 \\
\hline Alguém fuma dentro da casa & 218 & 52,8 ** & 195 & 47,2 & $<0,001$ \\
\hline A mãe fumou na gravidez? & 70 & 53,4 *夫 & 61 & 46,6 & 0,014 \\
\hline Peso de nascimento $<2.500 \mathrm{~g}$ & 61 & 51,7 & 57 & 48,3 & 0,055 \\
\hline O lactente já teve pneumonia & 142 & 77,6 ** & 41 & 22,4 & $<0,001$ \\
\hline Familiares com asma & 226 & 52,4 ** & 205 & 47,6 & $<0,001$ \\
\hline Familiares com alergia ou rinite alérgica & 188 & 48,5 & 200 & $51,5 * \star$ & 0,014 \\
\hline Familiares com alergia de pele & 109 & 49,5 & 111 & $50,5 * \star$ & 0,043 \\
\hline Resfriados no primeiro ano de vida (episódios) & & & & & $<0,001$ \\
\hline$<5$ & 172 & 30,1 & 400 & $69,9 * *$ & \\
\hline $5-9$ & 140 & 52,2 & 128 & 47,8 & \\
\hline 10 ou mais & 146 & $68,5 * \star$ & 67 & 31,5 & \\
\hline Idade no primeiro resfriado & & & & & $<0,001$ \\
\hline$<6$ meses & 63 & 26,3 & 177 & 73,8 ** & \\
\hline 6 meses ou mais & 378 & 49,2 & 390 & 50,8 & \\
\hline
\end{tabular}

* Teste qui-quadrado;

** Valores significantes.

avaliadas apresentaram pelo menos um episódio de chiado associado à infecção de vias respiratórias até os três anos de vida. Muiño et al. 23 acompanharam crianças desde o nascimento até os 12 anos e verificaram que $43 \%$ delas apresentaram sibilância até os quatro anos e não mais após, enquanto $6 \%$ apresentaram sibilância precoce que persistiu durante todo o seguimento. Mallol et al. 24, estudando crianças de até um ano de vida e com baixa condição socioeconômica, demonstraram que $80 \%$ delas haviam apresentado um ou mais episódios de sibilância. Esses autores sustentam que a exposição precoce e continuada a alérgenos ambientais pode ser um fator de risco nessa população. Porém, existem poucos estudos avaliando sibilância em lactentes, principalmente em países em desen- volvimento. Em parte, isso é causado pela falta de padronização nos métodos de coleta de dados ou pela dificuldade em se estabelecer o diagnóstico nessa faixa etária.

Quando analisado o questionário em sua totalidade, o gênero feminino foi o mais acometido quanto ao número de episódios de chiado, hospitalizações por bronquite ou pneumonia, diagnóstico médico de asma, tabagismo passivo e uso de corticosteroide oral (Tabela 1). Embora o sexo feminino tenha sido por nós identificado como fator de risco para sibilância recorrente, é o gênero masculino que tem sido demonstrado por vários pesquisadores como fator de risco para sibilância precoce 12,25 . Diferentemente do gênero masculino, o feminino tem sido associado à maior prevalência de asma no início da 
Fatores associados ao relato de sibilância no primeiro ano de vida em crianças com idades entre 12 e 15 meses, análise de regressão logística. Recife, Pernambuco, Brasil $(n=466)$.

\begin{tabular}{lccc}
\hline Variáveis & OR & IC95\% & Valor de $\mathbf{p}$ * \\
\hline Ter tido pneumonia & 4,01 & $2,64-6,04$ & $<, 001$ \\
Tabagismo no domicílio & 1,43 & $1,07-1,92$ & 0,016 \\
Familiares com asma & 1,73 & $1,30-2,30$ & $<, 001$ \\
Ter ido à creche & 1,55 & $1,12-2,13$ & 0,007 \\
Morar em local poluído & 1,51 & $1,14-2,01$ & 0,004 \\
Número de resfriados (episódios) & & $1,52-2,95$ & $<0,001$ \\
$\quad$ até 9 vs. 4 & 2,12 & $2,06-4,39$ & $<0,001$ \\
$>10$ vs. 4 & 3,01 & $1,28-2,61$ & 0,001 \\
Idade do primeiro resfriado (meses) & & 1,83 & \\
$\quad<6$ vs. $\geq 6$ & & & \\
\hline
\end{tabular}

IC95\%: intervalo de 95\% de confiança; OR: odds ratio.

* Teste qui-quadrado.

Fatores de risco associados à sibilância no primeiro ano de vida. Recife, Pernambuco, Brasil, 2007.

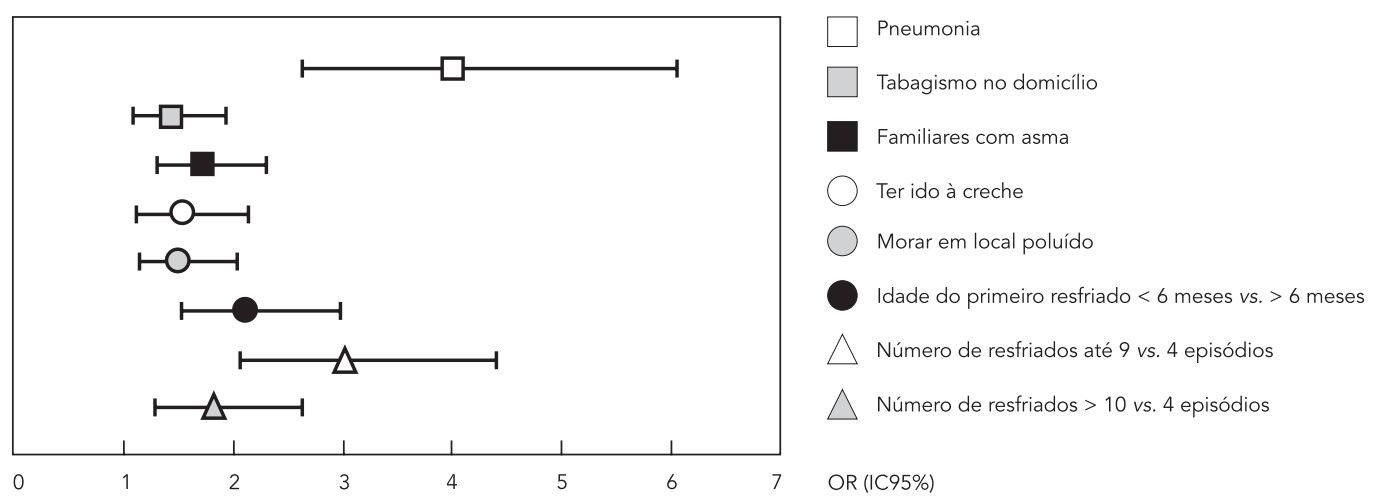

IC95\%: intervalo de 95\% de confiança; OR: odds ratio.

puberdade, principalmente entre meninas que apresentam puberdade precoce e obesidade 3,25 , além de estar associado a visitas em serviços de emergência por crises de asma e maior gravidade após os trinta anos 26. Será que o tabagismo passivo maior entre as meninas poderia ser outra explicação para a maior morbidade entre elas? Essa história a seguir é muito especulativa. Será que vale para nossa realidade? Foi demonstrado que as meninas são amamentadas por mais tempo que os meninos e que isto influenciaria no aparecimento de sibilância precoce, pois estes seriam apresentados mais cedo aos alimentos artificiais, contribuindo para a sensibilização precoce 9 . Entre os meninos o calibre das vias aéreas é menor quando comparado ao das meninas durante a infância, enquanto que durante a puberdade esta relação se inverte e isto favoreceria o aparecimento de sibilância nas adolescentes 27 . Outro ponto a considerar diz respeito à distribuição da população da cidade segundo o gênero. Há predomínio do sexo feminino. De fato, há mais mulheres do que homens em Recife (1,2:1) (Instituto Brasileiro de Geografia e Estatística. http://www. 
ibge.org.br, acessado em 07/Nov/2009). Porém, estudos de seguimento ou a repetição deste estudo no futuro será necessário para confirmar se as meninas apresentam mais sibilância que os meninos nesse local.

Entre as variáveis sociodemográficas e comportamentais analisadas, o grau de escolaridade materna, permanência em creche, poluição perto do domicílio, tabagismo durante a gestação e passivo após o nascimento, foram identificados como possíveis intervenientes para quadros de sibilância. Todos esses fatores normalmente estão associados ao baixo padrão socioeconômico, e consequentemente a agressões ambientais como o aumento do número de infecções respiratórias e maior exposição a alérgenos ambientais.

O tabagismo materno durante a gestação e o passivo (paterno) após o nascimento têm sido implicados no aparecimento precoce de sibilância 28. Demonstrou-se que em ambientes onde há fumantes, o risco para sibilância no terceiro ano de vida é três vezes maior ${ }^{3}$. Neste estudo, entre os sibilantes, o tabagismo materno durante a gestação foi de $15 \%$ e o tabagismo passivo, após o nascimento, $47 \%$, com um risco quase duas vezes maior, respectivamente, de a criança apresentar sibilância em ambiente de fumantes; dados semelhantes aos encontrados em outros estudos 29,30 que demonstram ainda a forte relação entre a persistência dos quadros de sibilância e a manutenção do tabagismo intradomiciliar. O tabagismo entre familiares foi associado a marcadores de atopia como IgE e interleucina (IL)-13. A IL-13 é uma citocina importante e está envolvida na produção de IgE e na patogênese da asma. A expressão de IL-13 em tecido pulmonar é associada à inflamação, secreção de muco, fibrose subepitelial e produção de eotaxina. O tabagismo durante a gestação está associado à produção aumentada de IL-13, e por isto acredita-se que parte dos efeitos decorrentes da exposição ao tabaco seriam mediados pela IL-13 e outras citocinas 31 .

Foi verificado alto consumo de corticosteroide oral pelas crianças pesquisadas, principalmente entre as meninas. Provavelmente, denote o fato de que essas crianças não tenham acompanhamento médico adequado, com falta de diagnóstico preciso, falhas no manuseio das crises de sibilância e visitas frequentes à emergência.

Entre as variáveis clínicas estudadas, verificamos que a história de asma entre familiares, passado de pneumonia, número de episódios de resfriados e idade precoce do primeiro episódio de resfriado (antes dos seis meses) foram fatores que propiciaram o aparecimento de sibilância. No nosso estudo, quase metade das crianças que apresentaram sibilância no primeiro ano de vida tinha familiares com asma, reforçando a observação de que a presença de história familiar de asma foi demonstrada como fator de risco para sibilância em pré-escolares e escolares 32 .

As infecções respiratórias virais são comuns na infância e também têm sido implicadas no desencadeamento de quadros de sibilância nos primeiros anos de vida e persistência de sintomas nos primeiros dez anos de idade 16,33. Resultados do Tucson Children's Respiratory Study demonstraram que o vírus respiratório sincicial acomete a maioria das crianças menores de dois anos, e que se associa a quadros de sibilância persistente que desaparece aos 13 anos 25. Garcia-Garcia et al. ${ }^{34}$, acompanhando crianças internadas por infecções respiratórias, demonstraram ser o metapneumovírus e o vírus respiratório sincicial os mais incriminados na persistência da sibilância aos três e cinco anos de idade.

Foi evidenciado que os níveis de IgE total em sangue de cordão umbilical de crianças que apresentaram infecções respiratórias sem sibilância não diferiam das que não apresentaram infecções respiratórias nos primeiros três anos, diferentemente das que apresentaram infecções respiratórias e sibilância associadas 3 . Crianças com infecções respiratórias de repetição apresentaram taxas elevadas de interferon gama aos nove meses de vida, indicando a pré-existência de fatores imunológicos no desencadeamento dos quadros de infecções respiratórias 35 .

Níveis elevados de IL-10 foram documentados em crianças em período de convalescença de infecção por vírus respiratório sincicial e isto foi associado ao risco para sibilância futura 36 . Porém, Ramsey et al. 37 relatam que, provavelmente, fatores não imunológicos como incremento da inflamação de vias aéreas ou a função pulmonar reduzida ao nascimento estariam envolvidos na maior susceptibilidade às infecções de vias aéreas inferiores. Provavelmente, alterações na função e/ou no tamanho das vias aéreas, a desregulação (congênita ou adquirida) do tônus da via aérea, alterações da resposta imunitária às infecções e variantes genéticas destas respostas seriam os fatores implicados nas infecções respiratórias e sibilância nessas crianças 37 .

Assim, neste estudo, demonstrou-se que ter resfriados em fase precoce da vida e em grande número, ter tido pneumonia e familiares com asma, exposição a tabaco no domicílio, ter ido à creche e residir em local poluído, estiveram associados à presença de quadros de sibilância no primeiro ano de vida em crianças frequentadoras de creches ou que procuravam atendimento nos postos de saúde da cidade de Recife. O conhecimento desses parâmetros é importante para que medidas preventivas sejam instituídas visando a 
reduzir a morbidade da sibilância nessa faixa etária, pois parte desse grupo provavelmente apresentará quadros de insuficiência respiratória ou diminuição da função pulmonar na adolescência ou quando adulto. Faz-se necessário o acompanhamento dessas crianças para comprovar se esses fatores de risco persistirão para o diagnóstico de asma no futuro.

\section{Resumo}

Verificar a prevalência de sibilância no primeiro ano de vida em lactentes da cidade do Recife, Pernambuco, Brasil e identificar fatores de risco a ela associados. Estudo realizado segundo o protocolo do Estudio Internacional de Sibilancia en Lactantes (EISL) em crianças com idades entre 12 e 15 meses. A amostra foi analisada segundo a presença ou não de sibilância. Foram entrevistados 1.071 pais por crianças com idades entre 12 e 15 meses. A prevalência de sibilância no primeiro ano de vida foi de 43\%, sem diferenças quanto aos gêneros. O relato de sibilância no primeiro ano de vida foi associado a ter tido pneumonia, ter familiares com asma, mais de nove episódios de infecções de vias aéreas superiores e primeiro episódio de resfriado antes de seis meses de idade $(p<0,001)$. A prevalência de sibilância no primeiro ano de vida entre lactentes da cidade do Recife é alta. Início precoce e número elevado de resfriados, ter familiares com asma e ter pneumonia foram fatores associados aos quadros de sibilância nessas crianças.

Sons Respiratórios; Asma; Lactente; Fatores de Risco

\section{Colaboradores}

Todos os autores contribuíram na elaboração do projeto, execução, análise dos dados e elaboração do artigo.

\section{Agradecimentos}

Aos alunos do curso médico da Universidade Federal de Pernambuco: Ana Tarsila Freitas, Carina Medeiros, Carla Tenório, Carla Urias, Gustavo Oliveira, Lara Ximenes, Raquel Coelho e Sabrina Perrusi pela dedicação constante na fase de coleta de dados. Às Secretarias de Saúde e de Educação da Prefeitura da Cidade do Recife pela colaboração. 


\section{Referências}

1. Wright AL. Epidemiology of asthma and recurrent wheeze in childhood. Clin Rev Allergy Immunol 2000; 22:33-44.

2. Csonka P, Kaila M, Laippal P, Kuusela A-L, Ashorn P. Wheezing in early life asthma at school age: predictors of symptom persistence. pediatr Allergy Immunol 2000; 11:225-9.

3. Taussing LM, Whight AL, Holberg CJ, Halonen M, Morgan WJ, Martinez FD. Tucson's children respiratory study: 1980 to present. J Allergy Clin Immunol 2003; 111:662-73.

4. Kotaniemi-Syrjänen A, Reijonen TM, Korhonen K, Korppi M. Wheezing requiring hospitalization in early childhood: predictive factors for asthma in six-year follow-up. Pediatr Allergy Immunol 2002; 13:418-25.

5. Akinbami LJ, Schoendorf KC. Trends in childhood asthma prevalence health care utilization, and mortality. Pediatrics 2002; 110:315-22.

6. Getahun D, Demissie K, Rhoads GG. Recent trends in asthma hospitalization and mortality in the United States. J Asthma 2005; 42:373-8.

7. von Mutius E, Nicolai T, Martinez FD. Prematurity as a risk factor for asthma in preadolescente children. J Pediatr 1993; 123:223-9.

8. Horak E, Morass B, Ulmer H. Association between environmental tobacco smoke exposure and wheezing disorders in Austrian preschool children. Swiaa Med Wkly 2007; 137:608-13.

9. Garcia-Marcos L, Mallol J, Solé D, Brand PL; The EISL Study Group. International study of wheezing in infants: risk factors in affluent and non-affluent countries during the first year of life. Pediatr Allergy Immunol 2010; 21:878-88.

10. van Merode T, Mass T, Twellaar M, Kester A, van Schayck CP. Gender-specific differences in the prevention of asthma-like symptoms in high-risk infants. Pediatr Allergy Immunol 2007; 18:196-200.

11. Panitch HB. The relationship between early respiratory viral infections and subsequent wheezing and asthma. Clin Pediatr 2007; 46:392-400.

12. Stein RT, Sherrill D, Morgan WJ, Holberg C, Halonen M, Taussing LM, et al. Respiratory syncicial vírus in early life and risk of wheeze na allergy by 13 years. Lancet 1999; 354:541-5.

13. Castro-Rodriguez JA. Assessing the risk of asthma in infants and pre-school children. Arch Bronconeumol 2006; 42:453-6.

14. Ly NP, Gold DR, Weiss ST, Celedon JC. Recurrent wheeze in early childhood and asthma among children at risk for atopy. Pediatrics 2006; 117:1132-8.

15. Mallol J, Andrade R, Auger F, Rodriguez J, Alvarado R, Figueroa L. Wheezing during the first year of life in infants from low-income population: a descriptive study. Allergol Immunopathol 2005; 33:257-63.

16. Mallol J, Garcia-Marcos L, Aguirre V, MartinezTorres A, Perez-Fernández V, Gallardo A, et al. The International Study of Wheezing in infants: questionnarie validation. Int Arch Allergy Immunol 2007; 144:44-50.

17. Asher MI, Keil U, Anderson HR, Baesley R, Crane J, Martinez FD, et al. International Study of Asthma and Allergies in childhood (ISAAC): rationale and methods. Eur Respir J 1995; 8:483-91.
18. Chong Neto HJ, Rosário NA; Grupo EISL Curitiba (Estudio Internacional de Sibilancias en Lactantes). Risk factors for wheezing in first year of life. J Pediatr (Rio J.) 2008; 84:495-502.

19. Chong Neto HJ, Rosário N, Dela Bianca AC, Solé D, Mallol J. Validation of a questionarie for epidemiologic studies of wheezing infants. Pediatr Allergy Immunol 2007; 18:86-7.

20. Dela Bianca AC, Wandalsen GF, Miyagi K, Camargo L, Cezarin D, Mallol J, et al. International Study of Wheezing in Infants (EISL): validation of written questionnaire for children aged below 3 years. J Investig Allergol Clin Immunol 2009; 19:35-42.

21. Chong Neto HJ, Rosário N, Solé D, Mallol J. Prevalência de sibilância recorrente em lactentes. J Pediatr (Rio J.) 2007; 83:357-62.

22. Martinez FD. What have we learned from the Tucson Children's Respiratory Study? Paediatr Respir Rev 2002; 3:193-7.

23. Muiño A, Menezes AMB, Reichert FF, Duquia RP, Chatkin M. Padrões de sibilância respiratória do nascimento até o início da adolescência: coorte de Pelotas (RS) Brasil, 1993-2004. J Bras Pneumol 2008; 34:347-55.

24. Mallol J, Andrade R, Auger F, Rodriguez J, Alvarado R, Figueroa L. Wheezing during the first year of life in infants from-income population: a descriptive study. Allergol Immunopathol (Madr) 2005; 33:257-63.

25. Alper Z, Sapan N, Ercan I, Canitez Y, Bilgel N. Risk factors for wheezing in primary school children in Bursa, Turkey. Am J Rhinol 2006; 20:53-63.

26. Skobrloff EM, Spivey WH, St Clair SS, Schoffstall JM. The influence of age and sex on asthma admissions. JAMA 1992; 268:3437-40.

27. de Marco R, Locatelli F, Sunyer J, Burney P. Differences in incidence of reported asthma related to age in men and women. A retrospective analysis of the data of the European Respiratory Health Survey. Am J Respir Crit Care Med 2000; 162:68-74.

28. Haberg SE, Stigum H, Nystad W, Nafstad P. Effects of pré and postnatal exposure to parental smoking on early chikdhood respiratory health. Am J Epidemiol 2007; 166:679-96.

29. Raherison C, Pénard-Morand C, Moreau D, Caillaud D, Charpin D, Kopfersmitt C, et al. In utero and childhood exposure to parental tobacco smoke, and allergies in schoolchildren. Respir Med 2007; 101:107-17.

30. Horak E, Morass B, Ulmer H. Association between environmental tobacco smoke exposure and wheezing disorders in Austrian preschool children. Swiss Med Wkly 2007; 137:608-13.

31. Sadeghnejad A, Karmaus W, Arshad SH, Kurukulaaratchy R, Huebner M, Ewart S. IL13 gene polymorphisms modify the effect of exposure to tobacco smoke on persistent wheeze and asthma in childhood, a longitudinal study. Respir Res 2008; 9:1-10.

32. Castro-Rodriguez JA, Holbreg CJ, Wright AL, Martinez FD. A clinical index to define risk of asthma in young children with recurrent wheezing. Am J Respir Crit Care Med 2000; 162:1403-6. 
33. Castro-Rodriguez JA, Holberg CJ, Wright Al, Halonen M, Taussig LM, Morgan WJ, et al. Association of radiologically ascertained pneumonia before age $3 \mathrm{yr}$ with asthmalike symptoms and pulmonary function during childhood: a prospective study. Am J Respir Crit Care Med 1999; 159:1891-7.

34. Garcia-Garcia ML, Calvo C, Perez-Breaza P, De Cea JM, Acosta B, Casas I. Prevalence and clinical characteristics of human metapneumovirus infections in hospitalized infants in Spain. Pediatr Pulmonol 2006; 41:863-71.

35. Copenhaver CC, Gern JE, Li Z, Shult PA, Rosenthal LA, Mikus LD, et al. Cytokine response patterns, exposure to viruses, and respiratory infections in the first year of life. Am J Respir Crit Care Med 2004; 1170:175-80.
36. van Bever HP, Desager KN, Hangendorens M. Critical evaluation of prognostic factors in childhood asthma. Pediatr Allergy Immunol 2002; 13:77-83.

37. Ramsey CD, Gold DR, Litonjua AA, Sredl DL, Ryan L, Celedon JC. Respiratory illnesses in early life and asthma and atopy in childhood. J Allergy Clin Immunol 2007; 119:150-7.

Recebido em 07/Jun/2010

Aprovado em 24/Mai/2011 